\title{
Symmetric Range Assignment with Disjoint MST Constraints
}

\author{
Eric Schmutz \\ Drexel University \\ Philadelphia, $\mathrm{Pa}$. \\ 19104,USA \\ Eric.Jonathan.Schmutz@drexel.edu
}

\begin{abstract}
If $V$ is a set of $n$ points in the unit square $[0,1]^{2}$, and if $R: V \rightarrow \Re_{+}$is an assignment of positive real numbers (radii) to to those points, define a graph $G(R)$ as follows: $\{v, w\}$ is an undirected edge if and only if the Euclidean distance $d(v, w)$ is less than or equal to $\min (R(v), R(w))$. Given $\alpha \geq 1$ and $k \in \mathbb{Z}_{+}$, let $R_{k}^{*}$ be the range assignment that minimizes the function $J(R)=\sum_{v \in V} R(v)^{\alpha}$, subject to the constraint that $G(R)$ has at least $k$ edge-disjoint spanning trees. For $n$ random points in $[0,1]^{2}$, the expected value of the optimum, $E\left(J\left(R_{k}^{*}\right)\right)$, is asymptotically $\Theta\left(n^{1-\frac{\alpha}{2}}\right)$. This is proved by analyzing a crude approximation algorithm that finds a range assignment $R_{k}^{a}$ such that the ratio $\frac{J\left(R_{k}^{a}\right)}{J\left(R_{k}^{*}\right)}$ is bounded.
\end{abstract}

\section{Categories and Subject Descriptors}

F.2.m [Analysis of Algorithms and Problem Complexity]: Miscellaneous

\section{General Terms}

Algorithms, Performance, Reliability, Theory

\section{Keywords}

Range assignment, probabilistic analysis, approximation algorithm, spanning tree

\section{INTRODUCTION}

If $V$ is a finite set of points $\Re^{2}$, and $R: V \rightarrow \Re_{+}$is an assignment of positive real numbers (radii) to the points, define an undirected graph $G(R)$ as follows. The vertex set is $V$, and $\{u, v\}$ is an undirected edge if and only if the Euclidean distance $d(u, v)$ is less than or equal to $\min (R(u), R(v))$. This is one of the standard models for connections between nodes of a wireless network: $R(v)$ represents the distance that node $v$ can effectively transmit, and the presence of an

Permission to make digital or hard copies of all or part of this work for personal or classroom use is granted without fee provided that copies are not made or distributed for profit or commercial advantage and that copies bear this notice and the full citation on the first page. To copy otherwise, to republish, to post on servers or to redistribute to lists, requires prior specific permission and/or a fee.

DialM-POMC'08, August 22, 2008, Toronto, Ontario, Canada.

Copyright 2008 ACM 978-1-60558-244-3/08/08 ...\$5.00. edge between two nodes indicates the availability of direct two-way communication between those nodes. For a suitable value of $\alpha$, the function $J(R)=\sum_{v \in V} R(v)^{\alpha}$ has been used as a measure of the energy spent by the nodes when transmitting. Hence it is a natural choice for an objective function to minimize. Some kind of connectivity constraint is needed to ensure that all pairs of nodes can communicate (either directly via an edge, or indirectly by forwarding messages). In this paper, the constraint imposed is the existence of $k$ edge-disjoint spanning trees. For positive integers $k>1$, and real numbers $\alpha \geq 1$, let $\Pi_{k, \alpha}$ be the following optimization problem.

$\Pi_{\mathbf{k}, \alpha}$ :

Instance: $V$, a finite set of points in $\Re^{2}$.

Objective: Choose $R: V \rightarrow \Re_{+}$so as to minimize

$$
J(R)=\sum_{v \in V} R(v)^{\alpha} .
$$

Constraint: $G(R)$ must have at least $k$ edge-disjoint spanning trees.

Given an instance of $\Pi_{k, \alpha}$, let $R_{k}^{*}$ denote an optimal choice of $R$. Thus $J\left(R_{k}^{*}\right)$ is the minimum feasible energy. The main result in this paper is an asymptotic estimate for the expected value of $J\left(R_{k}^{*}\right)$. For the stochastic model in which a random instance is selected by choosing $n$ points independently and uniform randomly from $[0,1]^{2}$, we prove that $E\left(J\left(R_{k}^{*}\right)\right)=\Theta\left(n^{1-\frac{\alpha}{2}}\right)$. More precisely,suppose that $\beta_{\alpha}$ is the "minimum spanning tree constant"[3], that $a<\frac{2}{5} \beta_{\alpha}$ and that $b>\left(2+\frac{2}{\alpha+2} k^{\alpha+2}\right) \beta_{\alpha}$. Then there is a positive constant $N_{k, \alpha}$ such that, for all $n>N_{k, \alpha}$, the expected value of $J\left(R_{k}^{*}\right)$ satisfies the inequalities

$$
a n^{1-\frac{\alpha}{2}} \leq E\left(J\left(R_{k}^{*}\right)\right) \leq b n^{1-\frac{\alpha}{2}} .
$$

One can regard $\Pi_{k, \alpha}$ as the restriction of a problem $\Pi$, in which both the the positive integer $k$ and the exponent $\alpha$ are given along with the points $V$ as input. The problem $\Pi$ is NP-hard, since it can be restricted to problems that are known to be $N P$-hard. In particular, for $k=1$, the existence of $k$ spanning trees is equivalent to connectivity. The problem of minimizing power, subject to the graph being connected, is known to be NP-hard [7] ,[8].I believe $\Pi_{k, \alpha}$ is NP-hard for general $k$ and $\alpha$, but have not yet completed a proof.

Several authors have considered range assignment problems with various other connectivity constraints. Vertex connectivity is paricularly important, and several authors 
have used this as a constraint. See for example, Lloyd et. al., Hajiaghayi et al., [10]Kortsarz , Mirrokni ,Nutov and Tsanko, [12] and the references therein, and Calinescu at.al [2],[5].The work of Blough et.al.[4]is closely related to ours because they estimated the expected value of the optimum power in the case $k=1$.

We should particularly mention $\tilde{\Pi}_{k, \alpha}=$ the symmetric range assignment problem with the constraint that $G$ has a $k$-edge-connected spanning subgraph. It is well-known [9] that any $2 k$-edge-connected graph has $k$ disjoint spanning trees. Hence any approximation algorithm for $\tilde{\Pi}_{2 k, \alpha}$ can, ipso facto, be used as an approximation algorithm for $\Pi_{k, \alpha}$. See Calinescu at.al [2],[5],Lloyd et.al.[13], and the work of Kortsarz et.al.,[12] and references therein for algorithms that use edge-connectivity as a constraint for range assignments. It is important to note that a graph with $k$ disjoint spanning trees need not be $2 k$-edge connected. Hence the optimum solution for a $\Pi_{k, \alpha}$ instance may have less power than the lowest power $2 k$-edge-connected range assignment for that instance. It follows that the worst case ratio of an algorithm for $\tilde{\Pi}_{2 k, \alpha}$ does not apply when the algorithm is used as an approximation algorithm for $\Pi_{k, \alpha}$.

\section{MINIMUM SPANNING TREES}

In this section, some elementary inequalities will be used to bound the objective function $J$ from above and below by less complicated functions related to minimum spanning trees. Like many authors, we will use minimum spanning tree(s) and related inequalities to construct and analyze an approximation algorithm.

Let $K_{c}=K_{c}(V)$ denote the complete graph on $V$. For $v, w \in V$, define the cost of the edge $\varepsilon=\{v, w\}$ to be $W(\varepsilon)=$ $d(v, w)^{\alpha}$. Obviously $W$ depends on $\alpha$, but to limit notational clutter, we write $W$ instead of $W_{\alpha}$. Throughout this paper, $\alpha$ is a fixed positive constant that is greater than or equal to one. It is important to note that, for $\alpha>1, W$ may not satisfy the triangle inequality. More precisely, one can have vertices $v_{1}, v_{2}, v_{3}$ for which

$$
W\left(\left\{v_{1}, v_{3}\right\}\right)>W\left(\left\{v_{1}, v_{2}\right\}\right)+W\left(\left\{v_{2}, v_{3}\right\}\right) .
$$

For any spanning subgraph $H \subseteq K_{c}$, define the cost of $H$ to be $W(H)=\sum_{\varepsilon \in H} W(\varepsilon)$, the sum of the costs of of its edges. If $H$ is a spanning subgraph of $K_{c}$, let $\Delta=\Delta(H)$ be the maximum of the degrees of the vertices in the graph $H$, and define $F(H)=\sum_{v} \max _{w \sim v} d(w, v)^{\alpha}$, where the maximum is over all neighbors $w$ that $v$ has in $H$. Then we have

LEMma 1. For any spanning subgraph $H$ of $K_{c}$,

$$
\frac{2}{\Delta} W(H) \leq F(H) \leq 2 W(H)
$$

Proof. For any finite list of positive real numbers, the maximum of the numbers is trivially bounded from above by the sum of the numbers. Therefore

$$
F(H) \leq \sum_{v} \sum_{u \sim v} d(u, v)^{\alpha}=2 W(H) .
$$

For the lower bound, note that, for any finite list of real numbers, the maximum of the numbers is bounded below by the average of the numbers. Thus, for each vertex $v$,

$$
\max _{u \sim v} d(u, v)^{\alpha} \geq \frac{1}{\operatorname{degree}(v)} \sum_{u \sim v} d(u, v)^{\alpha} \geq \frac{1}{\Delta} \sum_{u \sim v} d(u, v)^{\alpha} .
$$

Summing over all vertices $v$, we get the lower bound in Lemma 1.

Let $S_{k}^{*}$ be a minimum cost union of $k$ disjoint spanning trees for $G\left(R_{k}^{*}\right)$. To apply Lemma 1, we also need the following lemma.

LEMma 2. For all vertices $v$,

$$
R_{k}^{*}(v)=\max _{u} d(u, v)
$$

where the maximum is over vertices $u$ for which $\{u, v\}$ is an edge of $S_{k}^{*}$.

Proof. From the definition of $S_{k}^{*}$ (as a minimum cost union of $k$ disjoint spanning trees for $G\left(R_{k}^{*}\right)$ ), it is clear that $S_{k}^{*}$ is a subgraph of $G\left(R_{k}^{*}\right)$. This, and the definition of $G\left(R_{k}^{*}\right)$, imply that, for any edge $\{u, v\}$ of $S_{k}^{*}$, we must have $d(u, v) \leq \min \left(R_{k}^{*}(u), R_{k}^{*}(v)\right)$. Thus $R_{k}^{*}(v) \geq d(u, v)$ for any vertex $u$ that is adjacent to $v$ in $S_{k}^{*}$,i.e.

$$
R_{k}^{*}(v) \geq \max _{u} d(u, v) .
$$

We must prove that the inequality (1) cannot be strict. We suppose $R_{k}^{*}(v)>\max _{u} d(u, v)$, and derive a contradiction. Define

$$
\tilde{R}(w)= \begin{cases}R_{k}^{*}(w) & , w \neq v \\ \max _{u} d(v, u) & , w=v\end{cases}
$$

where the maximum is over all vertices $u$ that are adjacent to $v$ in $S_{k}^{*}$. Note that $\tilde{R}$ is feasible; $G(\tilde{R})$ has $k$ disjoint spanning trees since it still contains $S_{k}^{*}$. But $J(\tilde{R})<J\left(R_{k}^{*}\right)$. This contradicts the minimality of $J\left(R_{k}^{*}\right)$ in the definition of $R_{k}^{*}$

Applying Lemma 1 to Lemma 2, we get

Corollary 3. If $\Delta=\Delta\left(S_{k}^{*}\right)$ is the maximum vertex degree in $S_{k}^{*}$, then

$$
\frac{2}{\Delta} W\left(S_{k}^{*}\right) \leq J\left(R_{k}^{*}\right) \leq 2 W\left(S_{k}^{*}\right)
$$

Let $S^{K_{c}}$ be minimum cost union of $k$ spanning trees for $K_{c}$. In general, $\left.W\left(S_{k}^{*}\right) \geq W\left(S^{K_{c}}\right)\right) \geq k W\left(T_{1}\right)$. It is well known that $K_{c}(V)$ has a minimum weight spanning tree $T_{1}$ whose maximum degree less than or equal to 5 . (See, for example, Lemma 7.2 of [14]It is not true for arbitrary weights, but is true for the weights $W(\{u, v\})=d(u, v)^{\alpha}$ in this paper.)

Hence Corollary 3 does yield a reasonable lower bound for $E\left(J\left(R_{k}^{*}\right)\right)$ in the special case when $k=1$, namely

$$
E\left(J\left(R_{1}^{*}\right)\right) \geq \frac{2}{5} E\left(W\left(T_{1}\right)\right) .
$$

The distribution of $W\left(T_{1}\right)$ has been intensively studied by probability theorists[1],[3],[11],[17].For this paper, we need only the expected value: there is a constant $\beta_{\alpha}$, called the "minimum spanning tree constant", such that

$$
E\left(W\left(T_{1}\right)\right)=\beta_{\alpha} n^{1-\frac{\alpha}{2}}(1+o(1)) .
$$

Unfortunately, we do not have any upper bounds on $\Delta=$ $\Delta\left(S_{k}^{*}\right)$ for $k>1$, and we can no longer use this method to deduce a lower bound. However it is worth observing that $R_{k}^{*} \geq R_{1}^{*}$, so that we at least have these crude lower bounds:

$$
\left.\left.J\left(R_{k}^{*}\right)\right) \geq \frac{2}{5} W\left(T_{1}\right)\right)
$$


and

$$
E\left(J\left(R_{k}^{*}\right)\right) \geq E\left(J\left(R_{1}^{*}\right)\right) \geq \frac{2}{5} \beta_{\alpha} n^{1-\frac{\alpha}{2}}(1+o(1)) .
$$

\section{APPROXIMATION ALGORITHM}

This section presents a method for selecting a (suboptimal) set of $k$ disjoint spanning trees for $K_{c}$. Then, in the following section, we estimate the expected value of the sum of the weights of the set of trees that it selects, and then use this number to derive an upper bound the expected optimum value $E\left(J\left(R_{k}^{*}\right)\right)$.

Let $T_{1}$ be an MST for $K_{c}$ having maximum degree less than 6. Fix a vertex $v_{1} \in V$ as the root, and define the level of each vertex $v$ as the number of edges on the unique path from $v$ to $v_{1}$ in the tree $T_{1}$. (Level 0 consists of the root vertex $v_{1}$.) Let $h$ be the maximum of the levels, and for $i=0,1,2, \ldots, h$, let $\mathcal{L}_{i}$ consists of those vertices at level $i$, i.e. the vertices $v$ for which the graph distance is $i$.

Let $f: V /\left\{v_{1}\right\} \rightarrow V$ be the following "successor"function: $f(v)=$ the first vertex after $v$ on the path from $v$ to $v_{1}$. If $f^{(t)}$ denotes $f$ composed with itself $t$ times, then the domain of $f^{(t)}$ is $\bigcup_{i \geq t} \mathcal{L}_{i}$. If $f^{(t)}(v)$ is a vertex in $\mathcal{L}_{j}$, then $v$ is a vertex in $\mathcal{L}_{t+j}$. Thus $\left\{v, f^{(t)}(v)\right\}$ is an edge of $K_{c}$ that joins vertices whose levels differ by exactly $t$. We use $f$ to define $k-1$ forests as follows. For $t=2, \ldots, k$, let $F_{t}$ consist of all edges of the form $\left\{v, f^{(t)}(v)\right\}$ where $v$ is a vertex in level $t$ or higher. Since the edges of $F_{t}$ join vertices that are separated by exactly $t$ levels, it is clear that the $k-1$ forests are edgedisjoint and also share no edge with $T_{1}$. We need to modify them slightly so that they are in fact spanning trees rather than forests.

For each $t$, let $\omega_{t}$ be the number of components that the forest $F_{t}$ has. Let $\tau_{t, j}, 1 \leq j \leq \omega_{t}$ be the component trees of $F_{t}$, and let $V_{t, j}$ be the vertex set of $\tau_{t, j}$. Also let $\rho_{t, j}$ be the root (lowest level vertex) of $\tau_{t, j}$. The idea will be to hook all these roots up with low level vertices in the largest of the $\tau_{t, j}$ 's.

Choose tmax,jmax such that $V_{t \max , j \max }$ has maximum cardinality, and let $\mathcal{I}=\left\{(t, j): 1 \leq j \leq \omega_{t}\right.$ and $1 \leq t \leq$ $k$ and $(t, j) \neq(\operatorname{tmax}, j \max )\}$. Let $Z$ consist of those vertices of $V_{t \max , j \max }$ whose level is less than or equal to $5^{k}$. Later we will verify that $|Z|>|\mathcal{I}|$. We can therefore choose a $1-1$ function $\phi$ from $\mathcal{I}$ to $Z$. Now define $T_{t}$ to be the tree that is obtained from $F_{t}$ by adjoining the edges $\left\{\rho_{t, j}, \phi(t, j)\right\}$ with $1 \leq j \leq \omega_{t}$, and $(t, j) \neq(\operatorname{tmax}, j \max )$.

The validity of our construction depended upon our assumption that $|\mathcal{I}|<|Z|$. To verify this fact, begin with the following observation. For each $\ell$, there are at most $5^{\ell}$ vertices in level $\ell$ (because the a maximum degree of $T_{1}$ is strictly less than 6). On the other hand, each root $\rho_{t, j}$ is at some level less than $t$. Hence $\omega_{t} \leq \sum_{\ell=0}^{t-1} 5^{\ell}<\frac{5^{t}}{4}$. and $|\mathcal{I}|=-1+1+\sum_{t=2}^{k} \omega_{t}<\frac{5^{k+1}}{16}$. Thus

$$
|\mathcal{I}|<5^{k}
$$

The size of the largest component is at least as large as the average component size, so $\left|V_{t \max , j \max }\right| \geq \frac{n}{\omega_{t \max }}>\frac{4 n}{5^{k}}$. If $n>\frac{25^{k}}{4}$, then

$$
\left|V_{t \max , j \max }\right|>5^{k}
$$

Combining (6) and (5), we get the desired result: $|Z|>|\mathcal{I}|$.

\section{ANALYSIS}

Let $S_{k}^{a}$ be the union of $k$ spanning trees that was constructed in the previous section. Define

$$
R_{k}^{a}(v)=\max d(u, v),
$$

where the maximum is over all vertices $u$ for which $\{u, v\}$ is an edge of $S_{k}^{a}$. We know that $R_{k}^{a}$ is feasible because $G\left(R_{k}^{a}\right)$ contains $S_{k}^{a}$. Therefore, by the definition of $R_{k}^{*}$, we must have

$$
J\left(R_{k}^{*}\right) \leq J\left(R_{k}^{a}\right)
$$

By Lemma 1,

$$
J\left(R_{k}^{a}\right) \leq 2 W\left(S_{k}^{a}\right)
$$

It follows from (7) and (8) that

$$
E\left(J\left(R_{k}^{*}\right)\right) \leq 2 E\left(W\left(S_{k}^{a}\right)\right) .
$$

Thus we must estimate the expected cost of the spanning trees that are constructed using method in the previous section.

The choice of spanning trees in the preceding section involved three phases:

- The first phase, in which the MST $T_{1}$ is found.

- The second phase, in which the forests $F_{t}$ are chosen.

- The third phases, in which the forests' trees' roots are linked to vertices in $V_{t \max , j \max }$

Let $C_{1, k}, C_{2, k}, C_{3, k}$ respectively be the costs of the edges added in these phases. Then by (9), we have

$$
E\left(J\left(R_{k}^{*}\right)\right) \leq 2 E\left(C_{1, k}\right)+2 E\left(C_{2, k}\right)+2 E\left(C_{3, k}\right) .
$$

We already cited an estimate for the first term (see equation 2 ), so our goal in this section is to prove upper bounds for the second and third terms in (10).

First we estimate $E\left(C_{2, k}\right)$. Suppose $c=\{w, z\}$ is an edge of $F_{t}$ and $e=\{x, y\}$ is an edge of the MST $T_{1}$. We say $c$ covers $e$, and write either $c \triangleright e$ or $e \triangleleft c$, if the unique path in $T_{1}$ from $w$ to $z$ includes the edge $e$. Note that every edge $c$ in $F_{t}$ covers exactly $t$ edges $e$ of $T_{1}$. In the special case $\alpha=1$ we can use the triangle inequality and write

$$
\begin{gathered}
\sum_{c \in F_{t}} W(c) \leq \sum_{c \in F_{t}} \sum_{e \triangleleft c} W(e) \\
=\sum_{e \in T_{1}} \sum_{c \in F_{t}, c \triangleright e} W(e) \leq t \sum_{e \in T_{1}} W(e) .
\end{gathered}
$$

Then, by summing on $t$ and then averaging, we get

$$
C_{2, k} \leq\left(\begin{array}{l}
k \\
2
\end{array}\right) W\left(T_{1}\right)
$$

and then

$$
E\left(C_{2, k}\right) \leq\left(\begin{array}{l}
k \\
2
\end{array}\right) E\left(W\left(T_{1}\right)\right)=\left(\begin{array}{l}
k \\
2
\end{array}\right) \beta_{1} n^{1 / 2}(1+o(1)) .
$$

Unfortunately, for $\alpha>1$, the triangle inequality does not hold and we need a cruder argument. Suppose $c=\{w, z\}$ is an edge of $F_{t}$ and that $w=x_{0}, x_{1}, x_{2}, \ldots, x_{t-1}, x_{t}=z$ are 
the vertices on the path in $T_{1}$ from $w$ to $z$. Since $d(\cdot, \cdot)$ does satisfy the triangle inequality, we have

$$
d(w, z) \leq \sum_{i=0}^{t-1} d\left(x_{i}, x_{i+1}\right) \leq t \max _{0 \leq i<t} d\left(x_{i}, x_{i+1}\right) .
$$

Therefore

$$
d(w, z)^{\alpha} \leq t^{\alpha} \max _{0 \leq i<t} d\left(x_{i}, x_{i+1}\right)^{\alpha} \leq t^{\alpha} \sum_{i=0}^{t-1} d\left(x_{i}, x_{i+1}\right)^{\alpha} .
$$

As before, we have

$$
\begin{gathered}
\sum_{c \in F_{t}} W(c) \leq \sum_{c \in F_{t}} t^{\alpha} \sum_{e \triangleleft c} W(e) \\
=t^{\alpha} \sum_{e \in T_{1}} \sum_{c \in F_{t}, c \triangleright e} W(e) \leq t^{\alpha+1} \sum_{e \in T_{1}} W(e) .
\end{gathered}
$$

By summing on $t$ and averaging, we get (for any $\alpha \geq 1$ ),

$$
E\left(C_{2, k}\right) \leq \frac{k^{\alpha+2}}{\alpha+2} E\left(W\left(T_{1}\right)\right)=\frac{k^{\alpha+2}}{\alpha+2} \beta_{\alpha} n^{1-\frac{\alpha}{2}}(1+o(1)) .
$$

The estimate for $C_{3, k}$ can be quite crude because only $O(1)$ edges are added in phase three. Let $M=\max d(u, v)$, where the maximum is over all edges $\{u, v\}$ of $T_{1}$.For each edge $\{r, v\}$ that is added in phase 3 , there is a path from $r$ to $v$ in $T_{1}$, consisting of at most $5^{k}+k$ edges of $T_{1}$. By the triangle inequality (for $\mathrm{d}$ ), we have $d(r, v) \leq\left(5^{k}+k\right) M$, and consequently

$$
W(\{r, v\}) \leq\left(5^{k}+k\right)^{\alpha} M^{\alpha} .
$$

Combining (5) with (14), we get

$$
C_{3, k} \leq 5^{k}\left(5^{k}+k\right)^{\alpha} M^{\alpha} .
$$

From the results in Penrose [15]we know that, with asymptotic probability one, $M<\left(\frac{\log n}{n}\right)^{\frac{1}{2}}$. Hence

$$
E\left(C_{3, k}\right)=O\left(\left(\frac{\log n}{n}\right)^{\frac{\alpha}{2}}\right)=o\left(n^{1-\frac{\alpha}{2}}\right) .
$$

Putting (2),(13), and (16) into (10), we get the main result:

TheOREM 4. If $b>\left(2+\frac{2}{\alpha+2} k^{\alpha+2}\right) \beta_{\alpha}$, then for all sufficiently large $n$,

$$
E\left(J\left(R_{k}^{*}\right)\right)<b n^{1-\frac{\alpha}{2}} .
$$

\section{WORST CASE RATIO}

In this section, a slight modification of the preceding arguments will be used to bound the ratio $\frac{J\left(R_{k}^{a}\right)}{J\left(R_{k}^{*}\right)}$ of the energy computed by the approximation algorithm to the optimal energy. The idea is that both the numerator and denominator are $\Theta\left(W\left(T_{1}\right)\right)$.

For the denominator, we have (3), namely

$$
J\left(R_{k}^{*}\right) \geq J\left(R_{1}^{*}\right) \geq \frac{2}{5} W\left(T_{1}\right) .
$$

For the numerator, recall that

$$
J\left(R_{k}^{a}\right) \leq C_{1, k}+C_{2, k}+C_{3, k}
$$

where

$$
C_{1, k}=W\left(T_{1}\right)
$$

and

$$
C_{2, k} \leq\left(\begin{array}{l}
k \\
2
\end{array}\right) W\left(T_{1}\right)
$$

Recall from (15) that $C_{3, k} \leq 5^{k}\left(5^{k}+k\right)^{\alpha} M^{\alpha}$. where $M$ is the length of the longest edge of the MST $T_{1}$. Obviously no edge of $T_{1}$ can be longer than $\sqrt{2}$, so a ridiculously crude bound for $M^{\alpha}$ is

$$
M^{\alpha}=M^{\alpha-1} M \leq \sqrt{2}^{\alpha-1} M \leq \sqrt{2}^{\alpha-1} W\left(T_{1}\right) .
$$

Hence

$$
C_{3, k} \leq 5^{k}\left(5^{k}+k\right)^{\alpha} \sqrt{2}^{\alpha-1} W\left(T_{1}\right) .
$$

Define a constant $B$ (depending on $k$ and $\alpha$, but not on $n$ ) by

$$
B=\frac{1+\left(\begin{array}{c}
k \\
2
\end{array}\right)+5^{k}\left(5^{k}+k\right)^{\alpha} \sqrt{2}^{\alpha-1}}{2 / 5} .
$$

Putting (18),(19), and (20) into (17), and then using (3), we get

THEOREM $5.1 \leq \frac{J\left(R_{k}^{a}\right)}{J\left(R_{k}^{*}\right)}<B$.

\section{DISCUSSION}

The method for selecting spanning trees in section 3 is constructive, but it clearly inferior to known algorithms for finding a minimum weight set of $k$ disjoint spanning trees [6][16].I chose this method as an analytical device because it simplified the estimation of $E\left(J_{k}^{*}\right)=$ the average value of the optimum power $J_{k}^{*}$. However, I was not able to evaluate the limit $\lim _{n \rightarrow \infty} E\left(J_{k}^{*}\right) n^{\frac{\alpha}{2}-1}$. Presumably the limit exists for all $\alpha \geq 1$, but even that fact has not been proved.

\section{REFERENCES}

[1] D. Aldous and J. M. Steele. Asymptotics for Euclidean minimal spanning trees on random points. Probab. Theory Related Fields, 92(2):247-258, 1992.

[2] E. Althaus, G. Calinescu, I. I. Mandoiu, S. Prasad, N. Tchervenski, and A. Zelikovsky. Power efficient range assignment for symmetric connectivity in static ad hoc wireless networks. Wirel. Netw., 12(3):287-299, 2006.

[3] F. Avram and D. Bertsimas. The minimum spanning tree constant in geometrical probability and under the independent model: a unified approach. Ann. Appl. Probab., 2(1):113-130, 1992.

[4] D. M. Blough, M. Leoncini, G. Resta, and P. Santi. On the symmetric range assignment problem in wireless ad hoc networks. In TCS '02: Proceedings of the IFIP 17th World Computer Congress - TC1 Stream / 2nd IFIP International Conference on Theoretical Computer Science, pages 71-82, Deventer, The Netherlands, The Netherlands, 2002. Kluwer, B.V.

[5] G. Calinescu and P. jun Wan. Range assignment for biconnectivity and k-edge connectivity in wireless ad hoc networks. Mob. Netw. Appl., 11(2):121-128, 2006.

[6] J. Clausen and L. A. Hansen. Finding $k$ edge-disjoint spanning trees of minimum total weight in a network: an application of matroid theory. Math. Programming Stud., (13):88-101, 1980. Combinatorial optimization, II (Proc. Conf., Univ. East Anglia, Norwich, 1979). 
[7] A. E. F. Clementi, P. Penna, and R. Silvestri. On the power assignment problem in radio networks. Mob. Netw. Appl., 9(2):125-140, 2004.

[8] B. Fuchs. On the hardness of range assignment problems. Networks., 9999(9999), 2008.

[9] D. Gusfield. Connectivity and edge-disjoint spanning trees. Inform. Process. Lett., 16(2):87-89, 1983.

[10] M. Hajiaghayi, N. Immorlica, and V. S. Mirrokni. Power optimization in fault-tolerant topology control algorithms for wireless multi-hop networks. In MobiCom '03: Proceedings of the 9th annual international conference on Mobile computing and networking, pages 300-312, New York, NY, USA, 2003. ACM.

[11] H. Kesten and S. Lee. The central limit theorem for weighted minimal spanning trees on random points. Ann. Appl. Probab., 6(2):495-527, 1996.

[12] G. Kortsarz, V. S. Mirrokni, Z. Nutov, and E. Tsanko. Approximating minimum-power degree and connectivity problems. In LATIN, pages 423-435, 2008.
[13] E. L. Lloyd, R. Liu, M. V. Marathe, R. Ramanathan, and S. S. Ravi. Algorithmic aspects of topology control problems for ad hoc networks. Mob. Netw. Appl., 10(1-2):19-34, 2005.

[14] C. Monma and S. Suri. Transitions in geometric minimum spanning trees. Discrete Comput. Geom., 8(3):265-293, 1992. ACM Symposium on Computational Geometry (North Conway, NH, 1991).

[15] M. D. Penrose. The longest edge of the random minimal spanning tree. Ann. Appl. Probab., 7(2):340-361, 1997.

[16] J. Roskind and R. E. Tarjan. A note on finding minimum-cost edge-disjoint spanning trees. Math. Oper. Res., 10(4):701-708, 1985.

[17] J. M. Steele. Probability theory and combinatorial optimization, volume 69 of CBMS-NSF Regional Conference Series in Applied Mathematics. Society for Industrial and Applied Mathematics (SIAM), Philadelphia, PA, 1997.

@ ACM (2008) This is the author's version of the work. It is posted here by permission of ACM for your personal use. Not for redistribution. 